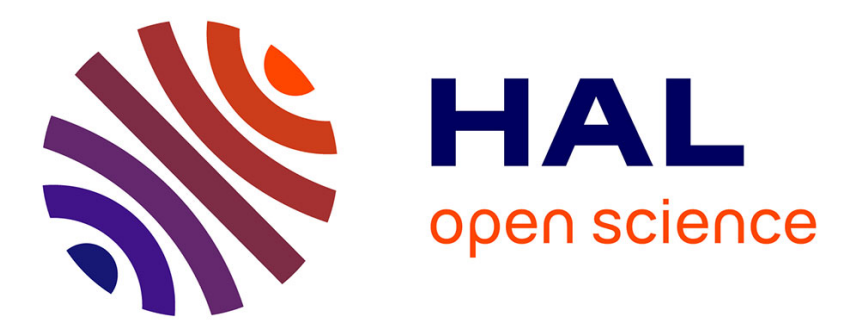

\title{
Learn with Diversity and from Harder Samples: Improving the Generalization of CNN-Based Detection of Computer-Generated Images
}

Weize Quan, Kai Wang, Dong-Ming Yan, Xiaopeng Zhang, Denis Pellerin

\section{- To cite this version:}

Weize Quan, Kai Wang, Dong-Ming Yan, Xiaopeng Zhang, Denis Pellerin. Learn with Diversity and from Harder Samples: Improving the Generalization of CNN-Based Detection of ComputerGenerated Images. Forensic Science International: Digital Investigation, 2020, 35, pp.301023:1-12. 10.1016/j.fsidi.2020.301023 . hal-02929038

\section{HAL Id: hal-02929038 \\ https://hal.science/hal-02929038}

Submitted on 3 Sep 2020

HAL is a multi-disciplinary open access archive for the deposit and dissemination of scientific research documents, whether they are published or not. The documents may come from teaching and research institutions in France or abroad, or from public or private research centers.
L'archive ouverte pluridisciplinaire HAL, est destinée au dépôt et à la diffusion de documents scientifiques de niveau recherche, publiés ou non, émanant des établissements d'enseignement et de recherche français ou étrangers, des laboratoires publics ou privés. 


\title{
Learn with Diversity and from Harder Samples: Improving the Generalization of CNN-Based Detection of Computer-Generated Images
}

\author{
Weize Quan ${ }^{\mathrm{a}, \mathrm{b}, \mathrm{c}}$, Kai Wang ${ }^{\mathrm{c}}$, Dong-Ming Yan ${ }^{\mathrm{a}, *}$, Xiaopeng Zhang ${ }^{\mathrm{a}}$, Denis \\ Pellerin ${ }^{\mathrm{C}}$ \\ ${ }^{a}$ Institute of Automation, Chinese Academy of Sciences, NLPR, 100190 Beijing, China \\ ${ }^{b}$ School of Artificial Intelligence, UCAS, 100049 Beijing, China \\ ${ }^{c}$ Univ. Grenoble Alpes, CNRS, Grenoble INP, GIPSA-lab, 38000 Grenoble, France
}

\begin{abstract}
Advanced computer graphics rendering software tools can now produce computergenerated $(\mathrm{CG})$ images with increasingly high level of photorealism. This makes it more and more difficult to distinguish natural images (NIs) from CG images by naked human eyes. For this forensic problem, recently some CNN(convolutional neural network)-based methods have been proposed. However, researchers rarely pay attention to the blind detection (or generalization) problem, i.e., no training sample is available from "unknown" computer graphics rendering tools that we may encounter during the testing phase. We observe that detector performance decreases, sometimes drastically, in this challenging but realistic setting. To study this challenging problem, we first collect four high-quality CG image datasets, which will be appropriately released to facilitate the relevant research. Then, we design a novel two-branch network with different initializations in the first layer to capture diverse features. Moreover, we introduce a gradient-based method to construct harder negative samples and conduct enhanced training to further improve the generalization of CNN-based detectors. Experimental results demonstrate the effectiveness of our method in improving the performance for the challenging task of "blind" detection of CG images.
\end{abstract}

\footnotetext{
* Corresponding author

Email address: yandongming@gmail.com (Dong-Ming Yan)
} 
Keywords: Image forensics, computer-generated image, convolutional neural network, generalization, negative samples

\section{Introduction}

With the advances of image rendering techniques and the prevalence of $3 \mathrm{D}$ modeling software, now it is easy to create computer-generated $(\mathrm{CG})$ images of very high visual quality [1. Fig. 1 shows four groups (columns) of CG images, which are respectively rendered by Artlantis 2], Autodesk [3, Corona 4], and VRay [5] from left to right. It is difficult to identify whether the images were captured by digital cameras or produced by computer graphics rendering because they have very high level of photorealism. Although rendering techniques bring convenience to our daily life, they also potentially challenge forensic systems. Therefore, distinguishing computer-generated images from natural images (NIs) has become an important research problem in image forensics. Hereafter, we call it as the CG image forensic problem.
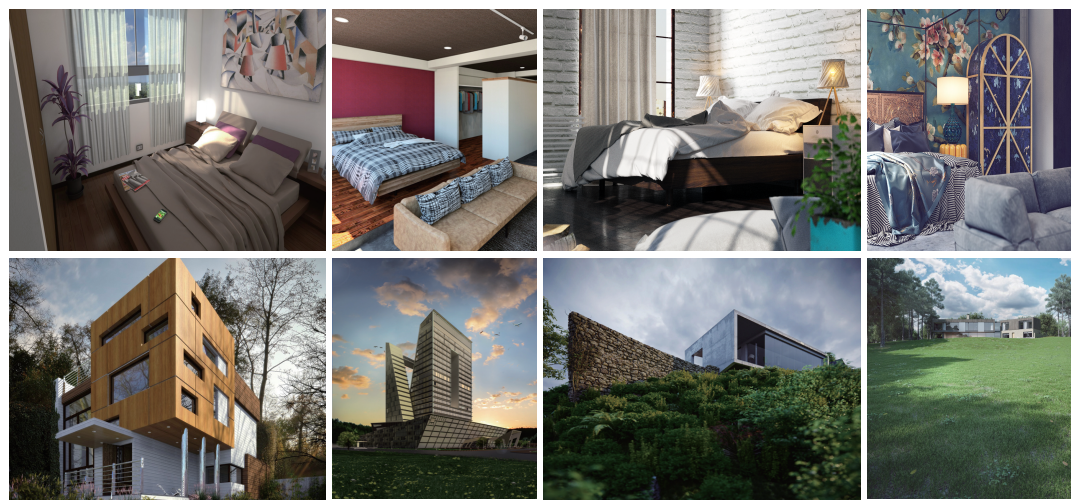

Figure 1: From left to right: four groups (columns) of computer-generated images which were rendered by Artlantis [2], Autodesk [3], Corona [4], and VRay [5], respectively.

In the past decades, researchers have proposed hand-crafted-feature-based methods [6, 7, 8, 9, 10, 11, 12 and CNN(convolutional neural network)-based methods 13, 14, 15, 16, 17, 18, 19] to solve this forensic problem. The former usually consists of multidimensional feature extraction and classifier training, 
and the latter directly formulates an optimization problem to find a good mapping function from a given image to its label and solves it efficiently in an end-to-end manner. Due to the powerful learning capacity of CNN, the CNNbased methods often achieve better forensic performance; however, the blind detection problem (or the so-called generalization problem) has been omitted in existing methods. This problem occurs when we train a CNN model using CG images from "known" computer graphics rendering techniques, and then test the model on images generated by "unknown" rendering techniques. Take Fig. 1 as an example, the CG images in second, third, and fourth columns (rendered by Autodesk, Corona, and VRay, respectively) are misclassified as NI by a CNN model trained on NIs and CG images rendered by Artlantis (first column). The misclassification is probably due to the existence of subtle and different "intrinsic" traces left by each rendering technique, e.g., in color use and light-material interaction. It is worth mentioning that this problem can be frequently encountered in practice when deploying detectors of CG images in real-world applications, as there can always exist CG images generated by new and/or customized rendering tools.

To improve the forensic performance, especially the generalization capability, we make efforts in two aspects of CNN: network architecture and network training. The core idea is to design and implement CNN with more diversity in feature learning and with the use of harder negative samples in the so-called enhanced training. Here, the negative sample means the artificially constructed image by only using the original training dataset (potentially combined with information from CNN model), and its ground-truth label is same as that of CG image. Specifically, we design a two-branch neural network which can capture more diverse features. Then, after the normal training, we generate the harder negative samples based on gradient information of CNN model, and then conduct enhanced training with original training dataset and additional negative samples.

Our contributions are summarized as follows: 
- We first raise and study in the literature the generalization issue of the CG image forensic problem. For the experimental study of this generalization problem, we collect four computer graphics datasets which were generated by four different rendering tools.

- We design a new network which has better generalization. The beginning part of the network has two branches with different initializations for the first layer.

- We propose a novel and effective model-centric method to generate negative samples. Given a trained model and a CG image, we iteratively modify this image via gradient-based distortion to make the distorted version close to the decision boundary of the CNN model. The gradient can be easily computed using backpropagation.

The rest of this paper is organized as follows. Section 2 reviews related work. Section 3 presents the datasets used for studying this generalization problem and for validating of our method. Section 4 discusses the motivation of our work, and elaborates the details of the proposed method. Section 5 evaluates the performance of our method. Section 6 draws the conclusions and discusses the future working directions.

\section{Related work}

\subsection{Distinguishing between NIs and CG images}

For the CG image forensic problem, there mainly exist two types of methods: hand-crafted-feature-based methods [6, 7, 8, 9, 10, 11, 12, and CNN-based methods [13, 14, 15, 16, 17, 18, 19.

Hand-crafted-feature-based methods usually consist of two separate phases: (1) designing discriminative features; (2) training classifiers, e.g., support vector machine (SVM). The final detection performance heavily depends on the discriminative capability of extracted features. Statistical methods are often applied to construct features, such as the first-order and higher-order wavelet 
statistics [7, 11], the statistical moments of wavelet characteristic function [8], the statistical property of local image edge patches [10], etc. The design of features is more or less based on the prior knowledge of human beings, e.g., potential differences between NI and CG images regarding the physical generation process [6] and texture [12], and in practice these features often exhibit limited forensic performance, especially on complex and challenging datasets [14].

Inspired by the notable success of CNN in the field of computer vision and pattern recognition, some recent works also applied CNN to solve the CG image forensic problem [13, 14, 15, 16, 17, 18, 19. Rahmouni et al. [13] explicitly extracted low-order statistical information of convoluted image as discriminative features and trained a model to distinguish computer graphics from photographic images. Quan et al. 14 proposed a CNN-based framework with two cascaded convolutional layers in the beginning to identify NIs and CG images. They also tried to understand what the deep model has learned about the differences between these two kinds of images by using a number of CNN visualization tools. Yao et al. [15] proposed a method based on sensor pattern noise and CNN to solve this task. In their method, they used several high-pass filters to enhance the residual signal as well as sensor pattern noise introduced by the digital camera device. He et al. [16] combined CNN and recurrent neural network (RNN) [20] to detect CG images. Nguyen et al. [17] used the capsule network [21] for the CG image forensic problem. Bhalang Tarianga et al. [18] proposed an attention-based recurrent model to classify computer-generated and natural images. Through exposing different characteristics between NIs and CG images using the channel and pixel correlation information, Zhang et al. 19] designed a hybrid-correlation-based CNN model.

While achieving decent classification accuracy on the detection of CG images, a very important issue, i.e., the generalization (or blind detection) problem, is rarely studied by prior works. Very recently, Quan et al. 22] considered the generalization problem in the task of colorized image detection, and proposed negative-sample-based enhanced training to effectively improve the generalization performance of CNN. In their work, they used linear interpolation of paired 
natural and colorized images to construct negative samples. Although this requirement of paired images is not satisfied for the classification of NIs and CG images, we extend this interpolation method in a straightforward way to the unpaired setting and find that it can still improve the CNN's generalization for the CG image forensic problem. This data-centric method only uses training images and is "blind" to the CNN model; therefore, motivated by a potential performance boost, we propose a new and more effective method (so-called model-centric method) by coupling the negative sample generation with the gradient information of CNN loss function. This model-centric method borrows idea from adversarial examples, and we briefly review this related topic in the next subsection.

\subsection{Adversarial examples}

In the following, we present some representative prior works on adversarial examples which are closely related to our method. Readers could refer to recent surveys 223, 24 for a comprehensive coverage of this rapidly evolving topic.

Szegedy et al. 25] found an intriguing phenomenon: several high-performance machine learning models, including advanced deep neural networks, are susceptible to adversarial examples (or adversarial attacks). When applying a small and imperceptible perturbation to a test sample, this perturbed version is very likely to be misclassified by trained deep models. Goodfellow et al. 26] explained that linearity in high-dimensional spaces is the primary cause of neural networks' vulnerability to adversarial perturbation. Based on this linear view, they proposed the fast gradient sign method (FGSM) to generate adversarial examples, where the required gradient can be computed efficiently using backpropagation. This gradient-based method is the backbone of many subsequent construction methods of adversarial examples. FGSM is essentially a one-step gradient-based method; therefore, a straightforward extension of this method is to apply it in a multiple-step fashion with smaller step size (in extreme cases, changing the value of each pixel only by 1 on each step) [27. Tramèr et al. [28] proposed to prepend FGSM by a small random step, which is based on the sign 
of a Gaussian distribution.

In computer vision and machine learning, adversarial examples have been used to improve the robustness of deep networks. There is less effort in the literature on using adversarial examples to improve network's generalization. To our knowledge, there is no such existing work in the image forensics community. In this paper, we propose a refined and appropriate method to generate adversarial examples as negative samples (i.e., simulated proxy of "unknown" CG images) for improving generalization. Specifically, our model-centric negative sample generation method is based on a new iterative version of FGSM, i.e., we randomly select certain percent of pixels to be changed by 1 for each step. The essential motivation of our method is to strictly control the strength of attack (in other words and loosely speaking, the location of negative samples relative to the decision boundary of $\mathrm{CNN}$ ). Our method has different original intention when compared to the conventional adversarial attacks in the field of machine learning, where they prefer to maximally cross the decision boundary with as small as possible perturbation. Our method shares some similarities with the iterative strategy of Tondi [29]; however, some differences exist: (1) 29] uses adversarial examples to carry out attacks, while we use adversarial examples to improve the generalization of CNN-based forensic detectors which is to our knowledge new in the literature. (2) With some technical choices, our iterative version is more finely controlled in terms of the confidence level of negative samples, which is important for the enhanced training.

\section{Datasets}

To study the generalization problem and validate our proposed method, we collect four CG dataset:푼. Artlantis [2], Autodesk [3], Corona [4], and VRay [5]. The CG images were downloaded from the websites of the four rendering software tools. The collected CG images have high level of photorealism and are

\footnotetext{
${ }^{1}$ The datasets, either images or download links, will be made available upon request.
} 
very close to real-world scenes. Some examples are shown in Fig. 1. The number of images of these four datasets are $1,620,1,620,1,593$, and 1,579, respectively. For each CG dataset, we randomly select 360 images as testing set, and the remaining images as training set (with the approximate ratio of 4:1). To guarantee the diversity of NIs, we combine two datasets of RAISE [30] and VISION 31] in our experiments. RAISE is a collection of 8,156 raw images that were taken at very high resolution and we randomly select 4,700 images. In order to simulate the real-world setting, we randomly resize and compress these raw images. For each raw image, we first resize with bicubic interpolation and with the length of its shorter edge as an integer randomly sampled from the set of $\{500,750,1000,1500,2000,2500,3000\}$. Then, we compress the resized image with quality factor randomly sampled from the range of $[70,100]$. VISION is composed of images captured by 35 mobile devices where each device includes 100 natural images (in total 3,500 images). In addition, these natural images were exchanged via the Facebook (high and low quality respectively) and WhatsApp social media platforms, and thus each image has four versions ("nat", "natFBH", "natFBL", and "natWA" in 31). Considering the same content of these four versions, we randomly select one version for each image and obtain 3,500 images. In the end, we have 8,200 NIs from RAISE and VISION.

In the following, we provide the details of datasets used in our experiments. We randomly select 5,040 NIs and duplicate approximately 4 times of each CG training set (5,040 CG images after duplication) to construct four final training datasets, corresponding respectively to the four rendering software tools (Artlantis, Autodesk, Corona, and VRay). From the remaining 3,160 NIs, we respectively select $360 \mathrm{NIs}$ for each CG dataset and combine corresponding testing set (360 CG images) to construct four final testing datasets. The remaining 1,720 NIs constitute the so-called natural validation dataset, which is used for the final CNN model selection in the stage of enhanced training (the details are described in Sec. 4.5. 


\section{Proposed method}

\subsection{Motivation}

For the CG forensic problem, current CNN-based approaches can achieve high classification accuracy. However, the performance of these forensic detectors often drops when testing the trained model on CG images generated by "unknown" computer graphics rendering tools. To solve this generalization problem, in this work, we consider two aspects of CNN: network architecture and network training. Our network design is inspired by Quan et al.' work 32 about the impact of CNN's first layer on forensic performance, where they proposed a simple criterion to combine the predictions of two independently trained networks for obtaining the final result. In our work, we design and implement a novel two-branch CNN model and apply different initialization strategy to the first layer of these two branches. This network can be trained in the end-to-end manner, and we expect to enrich the diversity of learned features through this ensemble-like design. For the network training, we adopt the enhanced training framework proposed in 22. An important component of enhanced training is the so-called negative samples, which are generated in [22] by linear interpolation of paired natural image and colorized image and which are deemed to be more difficult to classify. In this work, we propose two types of generation methods of negative samples (same label as CG images): (1) data-centric method, which constructs negative sample via linear interpolation of unpaired NI and CG image. (2) model-centric method, which generates negative sample by modifying the CG image based on the gradient of CNN model.

\subsection{Network architecture}

The standing point of our network design is to enrich the diversity of feature learning. Inspired by the observation in 32 and ensemble learning, we design a novel two-branch network to automatically and efficiently combine the kernels initialized with SRM (Spatial Rich Model) filters 33 and Gaussian random distribution in the beginning of network, and it can be trained in the standard 


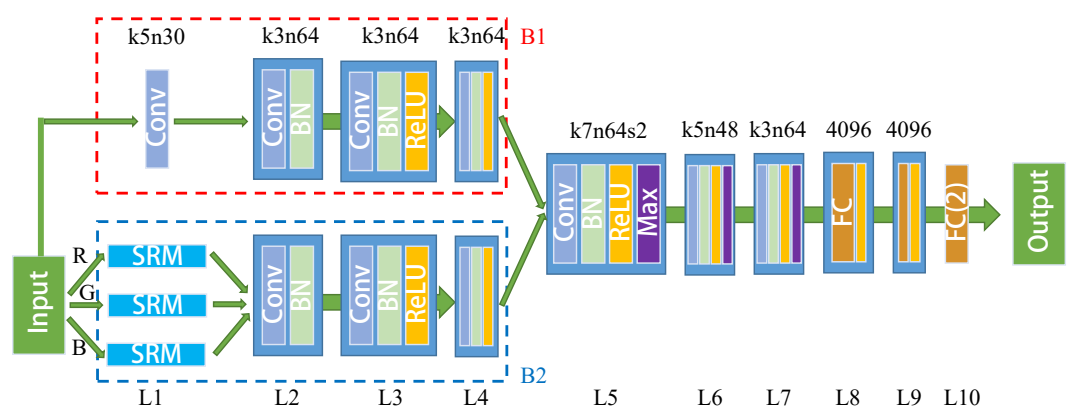

Figure 2: Architecture of our network named ENet. The network input is a $233 \times 233 \mathrm{RGB}$ image, and output is the class scores. For each convolutional layer, $\mathrm{k}$ is the kernel size, $\mathrm{n}$ is the number of feature maps, and $\mathrm{s}$ is the stride. " $\mathrm{FC}(2)$ " stands for a fully-connected layer with a 2-dimensional output of class scores. No padding exists in our network.

end-to-end way. This novel network is denoted by ENet (Ensemble Network), and the corresponding network architecture is shown in Fig. 2. In practice, our network takes the NcgNet proposed in 14] as backbone: the beginning part (from L1 to L4 in Fig. 2) has a new two-branch design; starting from L5, the network architecture is same as NcgNet. The input of ENet is an RGB image. After the first layer (so-called filter layer), we use three convolutional layers without pooling operation (L2-4) to analyze the filtered signal. The analysis results are concatenated (in the channel-wise manner) as the input of L5, and three consecutive convolutional layers (L5-7) and two fully-connected layers (L89) are applied to conduct high-level abstraction and reasoning. The last layer (L10) with softmax maps the high-level feature vector to the 2-dimensional class scores (NI and CG). In total, ENet is a 10-layer network.

In our network, from L2 to L4, we do not use any pooling operation so as to retain useful discriminative information, which also helps for improving the diversity between the two branches. In the second branch (B2 in Fig. 2), the SRM means that the convolutional kernels are fixed as the thirty $5 \times 5 \mathrm{SRM}$ residual filters borrowed from [33]. These three SRM blocks are applied to each color channel of input image, i.e., R, G, and $\mathrm{B}$, respectively. Then, all output channels are directly concatenated together to form a ninety-channel input of 
the second convolutional layer (L2). Except for the first layer (L1), each Conv is equipped with batch normalization (BN) layer. And we do not add activation layer after L2 to preserve useful information as much as possible. Following [14], all max-pooling layers have the same kernel size of $3 \times 3$ and a stride of 2 .

\subsection{Data-centric method}

This method constructs negative sample by linear interpolation, and the corresponding formulation is:

$$
I_{N S}=\alpha \cdot I_{N I}+(1-\alpha) \cdot I_{C G}
$$

where $I_{N S}$ is the negative sample, $I_{N I}$ is the natural image, $I_{C G}$ is the computergenerated image, and $\alpha \in\{0.1,0.2,0.3,0.4, \cdots, 0.9,0.99\}$ is the interpolation factor. For each factor, we randomly combine the NI and CG image of original training dataset. To clearly illustrate this process with examples, we select four CG images, and then randomly select an NI for each CG image to generate the negative sample via Eq. 1 with three different factors $(\alpha=0.1,0.5,0.9)$. The corresponding results are shown in Fig. 3. When $\alpha$ increases [from Fig. 34(c) to Fig. $3(\mathrm{e})]$, the negative samples are progressively getting closer to the natural images [Fig. 3(b)]. In addition, we allow the use of larger interpolation factor than the work in [22, i.e., $\alpha>0.4$, mainly due to the "blind" nature of this method, i.e., it is blind to the decision boundary of trained CNN model.

\subsection{Model-centric method}

This method is related to the gradient-sign-based adversarial sample generation. In this subsection, we first recall the formulation of FGSM [26] and its iterative variant [27. Then, we describe our iterative masked gradient sign method (IMGSM) for constructing negative sample.

Let $\mathbf{x}$ be the original (or clean) image, $\hat{\mathbf{x}}$ the perturbed version of $\mathbf{x}$ with expected target $t, \mathcal{M}$ a deep model and $J_{\mathcal{M}}(\mathbf{x}, t)$ the loss function (e.g., crossentropy loss) used to train the original model $\mathcal{M}$. The formulation of FGSM is

$$
\hat{\mathbf{x}}=\mathbf{x}-\epsilon \operatorname{sign}\left(\nabla_{\mathrm{x}} J_{\mathcal{M}}(\mathbf{x}, t)\right)
$$


(a)
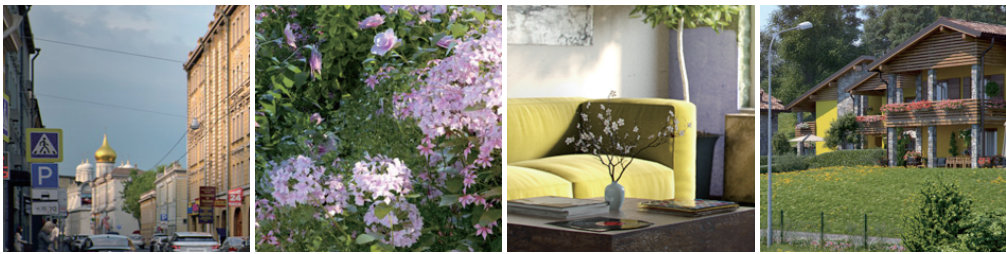

(b)
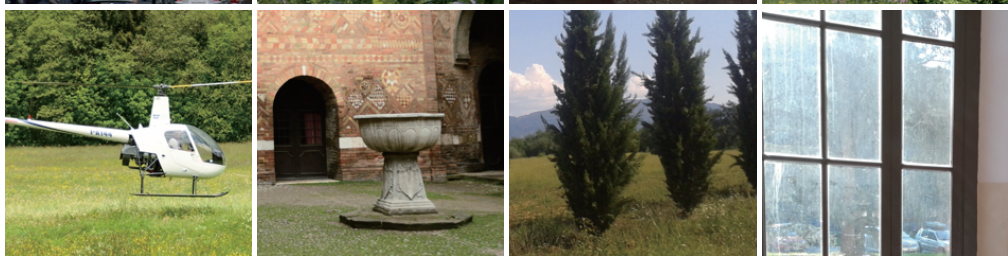

(c)
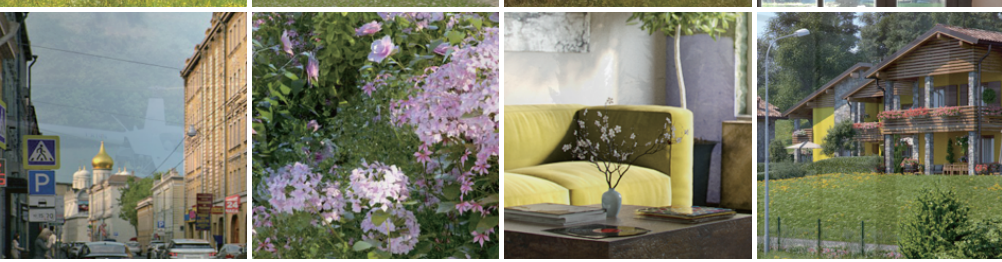

(d)
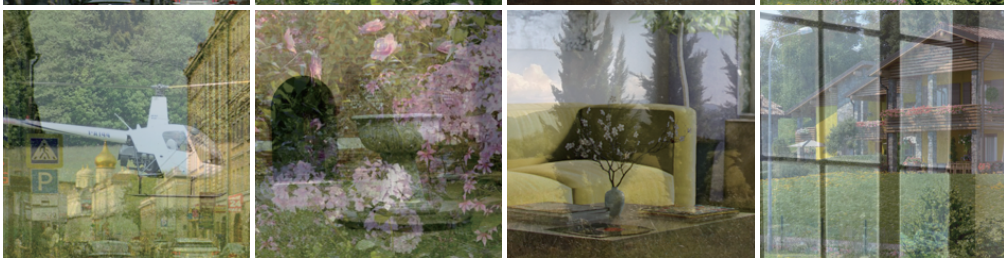

(e)
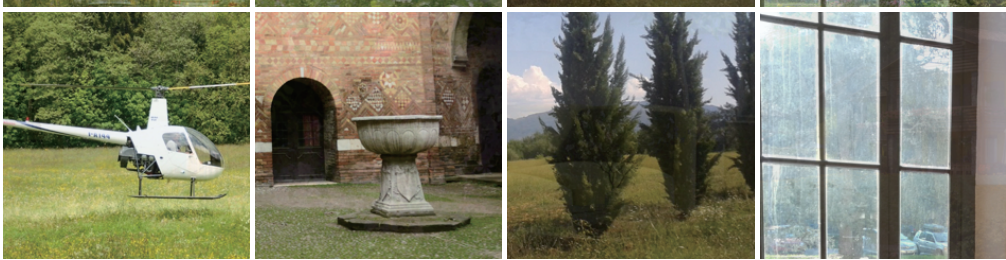

Figure 3: Examples of data-centric method. From top to bottom: (a) four CG images rendered by Corona [4]; (b) randomly selected NI for each CG image; (c), (d), and (e): the negative samples generated by Eq. 1 with CG image and NI in (a) and (b) of the same column, where the interpolation factor $\alpha$ is $0.1,0.5$, and 0.9 , respectively. We calculate the PSNR (peak signal-to-noise ratio) for the negative samples [(c), (d), and (e)], with the CG image [(a)] as the reference. PSNR values (in $\mathrm{dB}$ ) from left to right: (c) 28.12, 28.42, 28.09, and 27.32; (d) $14.24,14.44,14.31$, and 13.12; (e) 9.14, 9.33, 9.20, and 8.02. 
where hyper-parameter $\epsilon$ controls the magnitude of the perturbation.

An iterative variant of FGSM is

$$
\hat{\mathbf{x}}^{k+1}=C \operatorname{Cip} p_{\mathbf{x}, \epsilon}\left\{\hat{\mathbf{x}}^{k}-\beta \operatorname{sign}\left(\nabla_{\mathbf{x}} J_{\mathcal{M}}\left(\hat{\mathbf{x}}^{k}, t\right)\right)\right\},
$$

where $\operatorname{Clip}_{\mathbf{x}, \epsilon}\left\{\mathbf{x}^{\prime}\right\}$ is the operation which projects the image $\mathbf{x}^{\prime}$ into the $L_{\infty}$ $\epsilon$-neighbourhood of the source image $\mathbf{x}$ [27. Usually, the value of $\beta$ depends on the data type of image pixel (integer or float) and is set following the minimal distortion, i.e., changing the pixel value only by 1 for each modification.

Our goal is to modify a CG image $\mathbf{x}$ and output a harder negative sample $\hat{\mathbf{x}}$ with predicted probability $\mathbf{p}$ as NI under original trained model. $\hat{\mathbf{x}}$ plays the role of simulated proxy of "unknown" CG image that may be encountered during testing. To exactly control the predicted probability of negative sample, we introduce the iterative masked gradient sign method (IMGSM). Compared with Eq. 3, our formulation of IMGSM has two differences: (1) we have no clip operation because we mainly consider the attack confidence of negative sample (i.e., the probability $\mathbf{p}$ ) and do not need to strictly limit the magnitude of the perturbation. (2) we introduce the random-mask-based strategy to perturb the input image for each modification so that we can exactly control the attack confidence of negative sample, e.g., falling into a certain interval. The formulation is

$$
\hat{\mathbf{x}}^{k+1}=\hat{\mathbf{x}}^{k}-\beta \mathbf{m}_{\lambda} \odot \operatorname{sign}\left(\nabla_{\mathrm{x}} J_{\mathcal{M}}\left(\hat{\mathbf{x}}^{k}, t\right)\right),
$$

where $\odot$ is the element-wise product operation and $\mathbf{m}_{\lambda}$ is the binary mask whose elements are randomly set as zeroes with probability $\lambda$. Note that $\mathbf{x}, \nabla$, and $\mathbf{m}_{\lambda}$ have the same shape. In our experiment, the 8-bit integer pixel is scaled to float in $[-1,1]$, therefore, we set $\beta=2 / 255$ to guarantee the minimal distortion. In other words, after transforming back to the integer pixel value, $\pm \beta$ on float means adding or subtracting by 1 .

Algorithm 1 illustrates the process of model-centric negative sample generation. To constrain the predicted confidence of generated negative sample belonging to a certain interval $\left[\mathbf{p}_{\min }, \mathbf{p}_{\max }\right]$, we introduce adaptive strategy to adjust the mask probability $\lambda$ shown in line 14-18 of Algorithm 1. Starting 


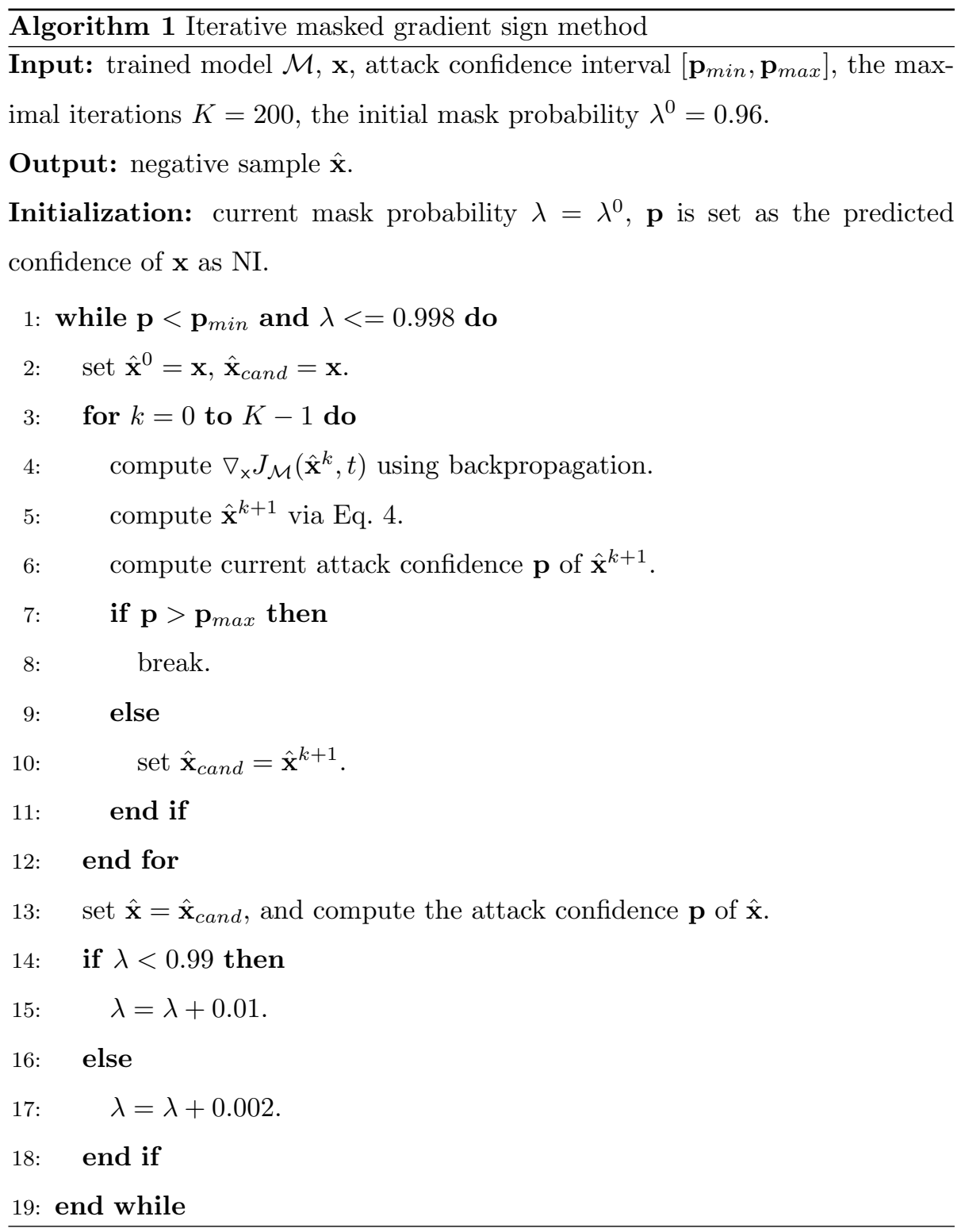




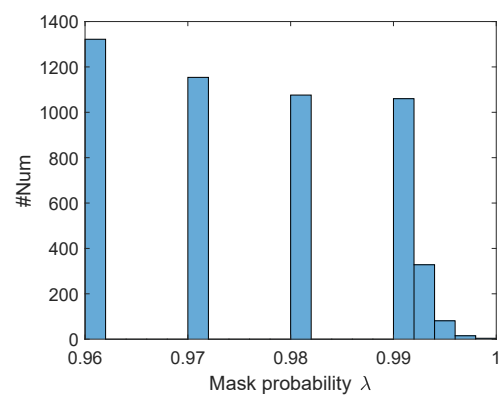

(a)

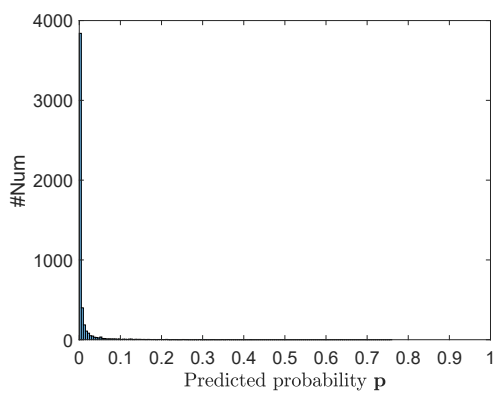

(c)

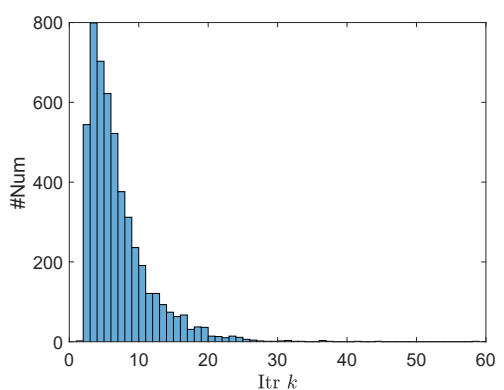

(b)

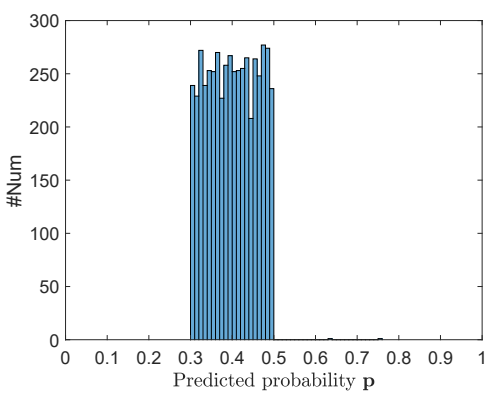

(d)

Figure 4: The statistics of model-centric negative sample generation: (a) mask probability $\lambda$, (b) iterations $k$, (c) the predicted probability $\mathbf{p}$ of original CG images, and (d) the predicted probability $\mathbf{p}$ of corresponding negative samples.

from a minimal value of $\lambda$ which experimentally leads to large distortion (thus large jump of $\mathbf{p}$ ) for each modification while iterating on $k$ in line 3 , the mask probability progressively increases when the predicted probability of generated negative sample cannot fall into the required interval. A mask with higher value of $\lambda$ leads to milder increase of $\mathbf{p}$ in each iteration, with more chance to meet the constraint of required interval for $\mathbf{p}$. In our experiment, we choose the confidence interval of generated negative sample as $[0.3,0.5]$, which means the negative sample is close to original classification boundary and in the side of CG image.

To clearly illustrate the process of model-centeric negative sample generation 

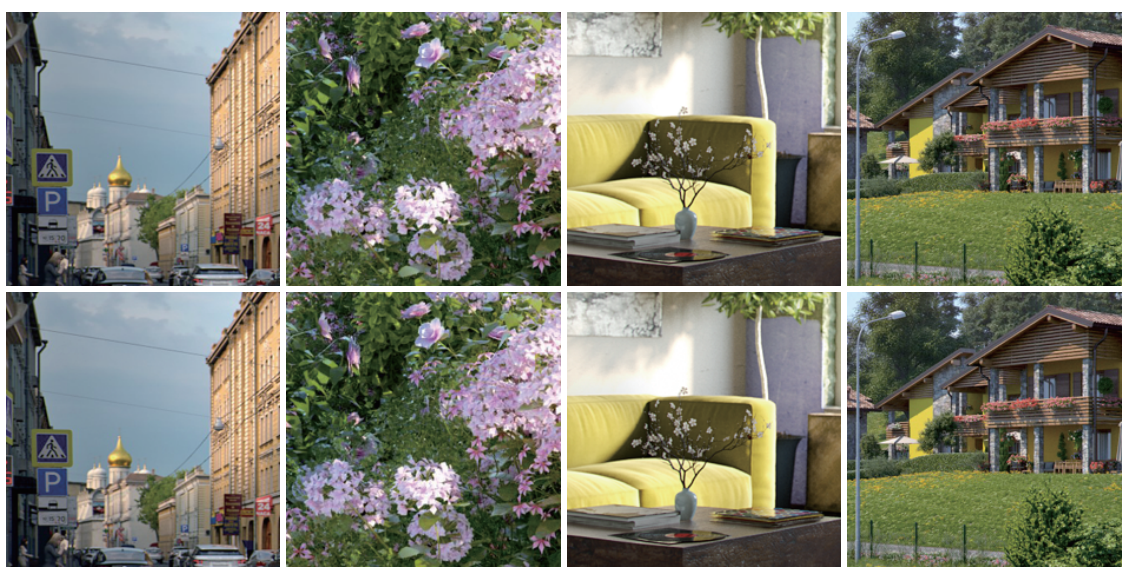

Figure 5: Four groups of CG sample (top row) and corresponding negative sample (bottom row) based on model-centric method. We calculate the PSNR for the negative sample, with the CG sample as the reference. PSNR values (in dB) from left to right: $56.32,58.92,56.63$, and 56.25 , respectively.

with an example, we train the ENet on Corona, and then generate the negative samples of CG images in training dataset (in total, 5040 images). Fig. 4 shows the histogram of mask probability $\lambda$ (a), iterations $k(\mathrm{~b})$, and predicted probability $\mathbf{p}$ of original CG images (c) and corresponding negative samples (d). Given a CG image, it is difficult to derive an algorithm which can theoretically guarantee that the predicted probability $\mathbf{p}$ of negative sample strictly falls into the expected interval (i.e., $[0.3,0.5]$ in our experiment). In practice, by using our adaptive mask strategy, we observe that experimentally almost all negative samples can successfully fall into this interval, like Fig. 4(d), where only two samples are not in $[0.3,0.5]$. This is because the two original CG images have $\mathbf{p}$ value larger than 0.5 , i.e., 0.76 and 0.64 .

In addition, Fig. 5 shows four groups of negative samples (bottom row) based on model-centric method and corresponding CG images (top row). From left to right: for CG sample, $\mathbf{p}=2.40 \mathrm{e}-5,4.92 \mathrm{e}-3,1.95 \mathrm{e}-4,2.01 \mathrm{e}-4$; for negative samples, $\mathbf{p}=0.32,0.38,0.48,0.35$. Comparing the two rows, we can find that the CG images and the corresponding negative samples are visually almost the 
same (see PSNR values in the caption of Fig. 57. Furthermore, compared with the PSNR values reported in the caption of Fig. 3 , those of Fig. 5 are obviously larger, which means that the perturbation introduced by model-centric method is much smaller. We also analyze the min/max perturbation for $\mathrm{CG}$ images in Fig. 5. i.e., the minimal/maximal perturbation value for pixels within an image. The min/max perturbations are from left to right: $-3 / 3,-3 / 2,-3 / 3$, and $-4 / 3$, respectively (pixel values are in the range of 0 to 255 ). These results demonstrate that when generating negative samples, gradient-based perturbation modifies very slightly the pixels of CG images in an almost imperceptible way.

\subsection{Network training}

In our work, a complete network training includes two stages: normal training and enhanced training. The CNN model first conducts normal training from scratch with the original training dataset $\mathcal{D}$, i.e., NIs and CG images generated by "known" rendering tools. After the model converges, we continue to train the model with enhanced training based on negative sample insertion. This enhanced training is an iterative process, and the main pipeline is described as follows.

- We construct negative samples using Eq. 1 or Algorithm 1, and insert them into $\mathcal{D}$.

- We update the parameters of $\mathcal{M}$ using $\mathcal{D}$. Starting from the second half of training process, we compute the error rate $r$ on the so-called natural validation dataset $\mathcal{V}$ and also mark the model as candidate model if its $r$ is less than $\theta$ (a threshold that determines the accepted degree of final classification accuracy on NIs; in our experiment, we set $\theta=4 \%$ ).

- The above two steps interleave until reaching the stop condition. If the error rates on $\mathcal{V}$ starting from the second half of training process are all larger than $\theta$, we stop the iteration process; otherwise, we stop when the number of iterations reaches maximal value $Z$ : for data-centric method, 
$Z=10$ ( $\alpha$ can take 10 values, see in Sec. 4.3); for model-centric method, $Z=20$.

- From all candidate models, we select the final model which has the maximal $r$.

\section{Experimental results}

\subsection{Implementation details}

All the experiments are implemented with PyTorch. The GPU version is GeForce ${ }^{\circledR}$ GTX 1080Ti of NVIDIA ${ }^{\circledR}$ corporation. In this work, we consider two recent state-of-the-art CNN models of YaoNet [15] and NcgNet [14. In [15], the images are first converted to grayscale and then fed into YaoNet to reduce the computational complexity. For convenience and fair comparisons, we directly use RGB images as the input of YaoNet and this variant achieves better performance compared with grayscale input. Following [14, all images in our experiments are resized using bicubic interpolation so that the shorter edge of each resized image has 512 pixels, and for each image, we rescale its pixel values to $[-1,1]$. The input size of network is $233 \times 233$. Stochastic gradient descent (SGD) with a minibatch of 32 is used to train ENet. For SGD optimizer, the momentum is 0.9 and the weight decay is $1 \mathrm{e}-4$. The initial learning rate is $1 \mathrm{e}-3$. For the normal training (only using original training dataset) of ENet, we divide the learning rate by 10 every 100 epochs, and the training procedure stops after 300 epochs. For the normal training of YaoNet and NcgNet, we follow the same learning rate schedule as described respectively in [15] and [14. In the stage of enhanced training of these three networks, we adopt the same strategy about learning rate: the learning rate is continued to be divided by 10 every 15 epochs (one insertion) and fixed after 4 iterations of negative sample insertion to avoid learning rate becoming too small. Following [14, we adopt the standard 10-crop testing [34: given a testing sample, the network extracts five patches of $233 \times 233$ pixels (the center and four corner patches), flips these five patches 
Table 1: The classification performance (HTER, in \%, lower is better) of different network architectures. "NcgNet_Comb" is the combination result of predictions of "NcgNet" and "NcgNet_SRM" according to the combination criterion used by 32 . For the sake of clarity, the results of generalization performance on "unknown" rendering engines are presented in italics.

\begin{tabular}{c||c|c|c|c}
\hline Network & Autodesk & Artlantis & Corona & VRay \\
\hline YaoNet & 4.61 & 28.14 & 15.00 & 21.17 \\
\hline NcgNet & 2.84 & 16.61 & 12.78 & 16.58 \\
\hline NcgNet_SRM & 2.42 & 17.97 & 8.97 & 16.09 \\
\hline NcgNet_Comb & 2.16 & 13.14 & 7.75 & 12.61 \\
\hline ENet & 1.56 & 10.39 & 7.67 & 13.39 \\
\hline
\end{tabular}

in the left-right direction (i.e., horizontal reflection), and then averages the predictions of total 10 patches as the final result. We employ the half total error rate (HTER) to evaluate the detection performance. The HTER is defined as the average of misclassification rates (in \%) of NIs and CG images, here same as the overall error rate on balanced testing datasets. In this work, all reported results are the average of 5 runs.

\subsection{Validation of our proposed network}

We validate our network architecture design of ENet in terms of the conventional classification performance and the generalization capability. All the networks are trained on Autodesk and tested on Artlantis, Corona, and VRay. The corresponding results are reported in Table 1. Compared with YaoNet, NcgNet and NcgNet_SRM, ENet demonstrates better performance for both "known" and "unknown" rendering engines. Here, the NcgNet_SRM is the variant of NcgNet where the first layer of NcgNet is replaced with three SRM blocks like the first layer of B2 in Fig. 2. In addition, although NcgNet_Comb and ENet have comparable results, the former needs to train two models separately, i.e., which inevitably and approximately doubles the number of parameters and training time.

Next, we evaluate the performance of four variants of our proposed ENet, 
Table 2: The classification performance (HTER, in \%, lower is better) of our proposed ENet and its four variants. All networks are trained on Autodesk. The results of generalization performance on "unknown" rendering engines are presented in italics.

\begin{tabular}{c||c|c|c|c}
\hline Network & Autodesk & Artlantis & Corona & VRay \\
\hline ENet_d & 2.00 & 15.06 & 10.25 & 16.11 \\
\hline ENet_SRM & 2.00 & 11.19 & 7.30 & 13.44 \\
\hline ENet_d_half & 1.72 & 12.53 & 8.44 & 13.44 \\
\hline ENet_rand & 2.28 & 15.97 & 10.33 & 16.89 \\
\hline ENet & 1.56 & 10.39 & 7.67 & 13.39 \\
\hline
\end{tabular}

i.e., ENet_d, ENet_SRM, ENet_d_half, and ENet_rand. We remove the B2 of ENet (the blue dotted rectangle in Fig. 2) and double the number of feature maps of L3 and L4 to obtain ENet_d. Similarly, we remove the B1 of ENet (the red dotted rectangle in Fig. 2) and also double the number of feature maps of L3 and L4 to obtain ENet_SRM. For ENet_d_half, we combine the three SRM blocks with the first layer of ENet_d, i.e., concatenating the outputs of these three SRM blocks with the output of first layer of ENet_d to form a 120channel input of the L2 of ENet_d. In addition, we use the Gaussian random distribution to initialize the first layer of B2 of ENet and make the corresponding ninety kernels trainable, and this replaces the B2's original setting of using fixed SRM filters. The corresponding model is denoted by ENet_rand. Note that, the number of parameters of these four variants are slightly larger than that of ENet. Among these five networks, the ENet achieves better overall performance (see Table 22. We find that ENet outperforms ENet_rand (comparing the row of "ENet_rand" and "ENet"). This demonstrates that our ensemble-like design, with different intializations at the first layer of the two branches, can improve the performance of network, especially generalization. It can also be observed that ENet_SRM and ENet_d_half have satisfying performance and that the ENet can further decrease the conventional classification error rate meanwhile slightly improving the overall generalization. Our conjecture is that convolutional layers without pooling operation in the front part of the two-branch structured ENet 


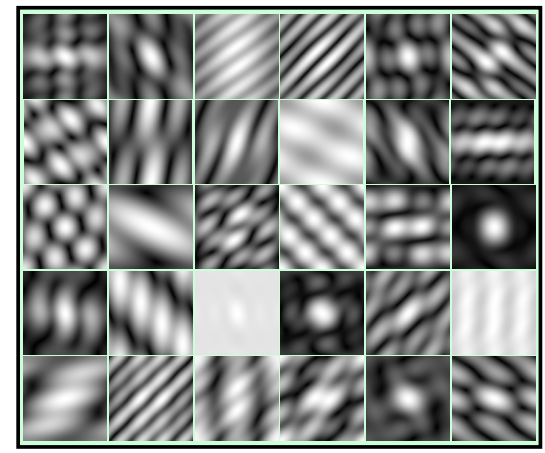

(a)

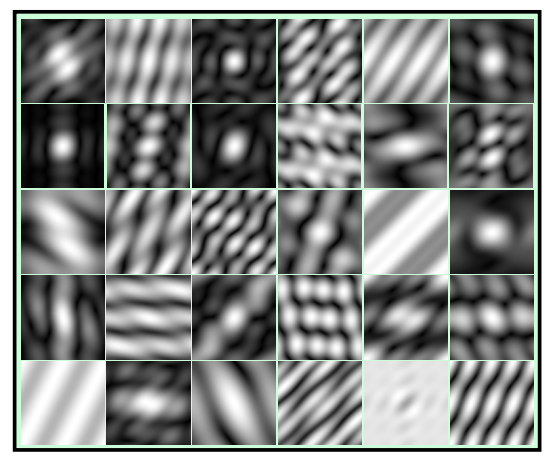

(c)

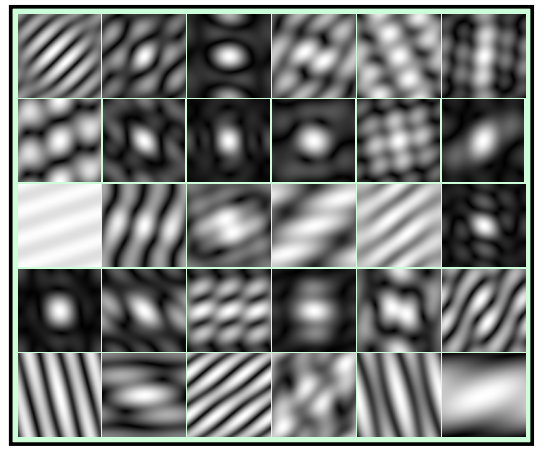

(b)

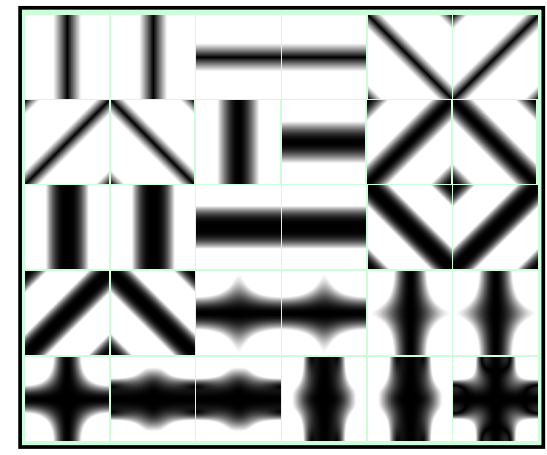

(d)

Figure 6: Visualization of FFT of the first-layer filters of ENet. B1: (a)[R], (b)[G] and (c)[B]; B2: (d) $[\mathrm{SRM}]$.

tend to preserve the diversity of the information flow, which may be helpful to improve the forensic performance.

Furthermore, we qualitatively analyze the first-layer filters of ENet from the signal processing perspective, i.e., visualizing the FFT (fast Fourier transform) of the first-layer kernels of two branches (B1 and B2 in Fig. 22), and the corresponding results are shown in Fig. 6. Different from the apparent highpass response in (d) for SRM, many kernels in the first layer of B1 capture the band-pass frequency information and several kernels even have relatively low-pass response. This implies that the ENet can automatically capture different frequency band information with the help of our ensemble-like design. 
This is beneficial to enrich the extracted features and to improve the detection performance.

\subsection{Effect of enhanced training}

In this work, we introduced two types of negative sample generation: datacentric method and model-centric method. To verify the effectiveness of these two methods for generalization improvement, we conduct extensive experiments with three CNN models on four datasets. We report in Table 3 , 4,5 and 6 the performance before (the column of "NT") and after (the columns of "ETI" and "ET-G") enhanced training of YaoNet, NcgNet, and ENet, when they are trained on Artlantis, Autodesk, Corona, and VRay, respectively. For each table, starting from the second column, each consecutive three columns form a group, in total four groups. The first group (e.g., "Artlantis" in Table 3 ) is the conventional classification error rate, and the remaining three groups (e.g., "Autodesk", "Corona", and "VRay" in Table 3) are the generalization performance.

From Table 36, we find that enhanced training with negative sample insertion based on data-centric and model-centric methods usually leads to slight increase of conventional classification error rate; however, the generalization of the three networks can be apparently and consistently improved by these two methods (except for one case in Table 5, when we trained YaoNet on Corona with data-centric method and tested on VRay, with a small increase of HTER by $1.39 \%$ from $9.14 \%$ to $10.53 \%$, but model-centric method can decrease it to 7.19\%). As an example of performance improvement, when we trained ENet on Artlantis with enhanced training based on model-centric method (comparing the columns of "NT" and "ET-G" of the last row in Table 3), the conventional classification accuracy decreases by $1.44 \%$, whereas the generalization is improved by $5.75 \%$ (Autodesk), $7.30 \%$ (Corona), and $6.36 \%$ (VRay), respectively. Furthermore, in Table 3 6 , the HTER value of ENet is always the lowest among three networks except for one case $(3.08 \%$ in Table 4 , i.e., training on Autodesk and testing on Autodesk with data-centric method). This illustrates our proposed 
Table 3: Performance (HTER, in \%, lower is better) of three networks when trained on Artlantis. "NT" stands for normal training; "ET-I" and "ET-G" stands for enhanced training with negative samples produced by unpaired linear interpolation and gradient-based distortion, respectively; The generalization performance results on "unknown" rendering engines are in italics (same for Table 4, 5 , and 6).

\begin{tabular}{|c|c|c|c|c|c|c|c|c|c|c|c|c|}
\hline \multirow{2}{*}{ Network } & \multicolumn{3}{|c|}{ Artlantis } & \multicolumn{3}{|c|}{ Autodesk } & \multicolumn{3}{|c|}{ Corona } & \multicolumn{3}{|c|}{ VRay } \\
\hline & NT & ET-I & $\overline{E T-G}$ & $\mathrm{NT}$ & ET-I & ET-G & NT & ET-I & ET-G & $\mathrm{NT}$ & ET-I & ET-G \\
\hline YaoNet & 3.86 & 5.17 & 3.94 & 20.31 & 12.61 & 12.64 & 18.17 & 14.14 & 13.72 & 15.11 & 13.42 & 12.47 \\
\hline NcgNet & 3.17 & 3.47 & 3.61 & 12.69 & 8.47 & 8.17 & 16.00 & 11.72 & 11.22 & 12.58 & 10.78 & 9.58 \\
\hline ENet & 1.31 & 2.31 & 2.75 & 10.06 & 5.09 & 4.31 & 14.58 & 8.15 & 7.28 & 11.86 & 7.22 & 5.50 \\
\hline
\end{tabular}

Table 4: Performance (HTER, in \%, lower is better) of three networks when trained on Autodesk

\begin{tabular}{|c|c|c|c|c|c|c|c|c|c|c|c|c|}
\hline \multirow{2}{*}{ Network } & \multicolumn{3}{|c|}{ Autodesk } & \multicolumn{3}{|c|}{ Artlantis } & \multicolumn{3}{|c|}{ Corona } & \multicolumn{3}{|c|}{ VRay } \\
\hline & $\mathrm{NT}$ & ET-I & $\overline{\text { ET-G }}$ & $\mathrm{NT}$ & ET-I & $\overline{\text { ET-G }}$ & $\mathrm{NT}$ & ET-I & $\overline{\text { ET-G }}$ & $\mathrm{NT}$ & ET-I & $\overline{\text { ET-G }}$ \\
\hline YaoNet & 4.61 & 3.86 & 2.72 & 28.14 & 22.50 & 16.84 & 15.00 & 11.31 & 8.00 & 21.17 & 16.00 & 12.97 \\
\hline NcgNet & 2.84 & 2.87 & 2.67 & 16.61 & 11.48 & 11.03 & 12.78 & 9.50 & 9.06 & 16.58 & 12.32 & 12.64 \\
\hline ENet & 1.56 & 3.08 & 2.39 & 10.39 & 5.64 & 5.58 & 7.67 & 5.39 & 4.86 & 13.39 & 7.47 & 8.22 \\
\hline
\end{tabular}

Table 5: Performance (HTER, in \%, lower is better) of three networks when trained on Corona.

\begin{tabular}{|c|c|c|c|c|c|c|c|c|c|c|c|c|}
\hline \multirow{2}{*}{ Network } & \multicolumn{3}{|c|}{ Corona } & \multicolumn{3}{|c|}{ Artlantis } & \multicolumn{3}{|c|}{ Autodesk } & \multicolumn{3}{|c|}{ VRay } \\
\hline & $\mathrm{NT}$ & ET-I & $\overline{E T-G}$ & $\mathrm{NT}$ & ET-I & $\overline{E T-G}$ & $\mathrm{NT}$ & ET-I & $\overline{E T-G}$ & $\mathrm{NT}$ & ET-I & $\overline{E T-G}$ \\
\hline Yao & 3.79 & 3.79 & 28 & 9.53 & 17.17 & .00 & 10.87 & 10.50 & 8.42 & .14 & 10.53 & 7.1 \\
\hline $\mathrm{Nc}$ & 73 & 3.06 & 81 & 74 & 5 & 3.69 & 9.56 & 7.25 & 7.67 & 8.08 & 6.11 & 5.75 \\
\hline ENet & 1.50 & 2.72 & 2.14 & 16.08 & 7.61 & 6.39 & 7.92 & 6.83 & 7.03 & 7.78 & 4.36 & 4.39 \\
\hline
\end{tabular}

Table 6: Performance (HTER, in \%, lower is better) of three networks when trained on VRay.

\begin{tabular}{|c|c|c|c|c|c|c|c|c|c|c|c|c|}
\hline \multirow{2}{*}{ Network } & \multicolumn{3}{|c|}{ VRay } & \multicolumn{3}{|c|}{ Artlantis } & \multicolumn{3}{|c|}{ Autodesk } & \multicolumn{3}{|c|}{ Corona } \\
\hline & NT & ET-I & ET-G & NT & ET-I & ET-G & NT & ET-I & ET-G & NT & ET-I & ET-G \\
\hline YaoNet & 4.28 & 4.22 & 3.64 & 16.77 & 15.75 & 11.64 & 15.30 & 11.89 & 9.64 & 9.05 & 7.47 & 6.33 \\
\hline NcgNet & 3.20 & 3.53 & 2.84 & 14.06 & 10.19 & 8.97 & 15.00 & 8.86 & 8.53 & 5.53 & 5.17 & 5.33 \\
\hline ENet & 1.25 & 2.22 & 1.72 & 11.58 & 6.94 & 5.39 & 9.97 & 5.97 & 6.08 & 4.53 & 4.31 & 3.17 \\
\hline
\end{tabular}

ENet has the superior performance.

We also compare the performance of data-centric and model-centric based enhanced training with that of "mixup" 35. "mixup" is a learning principle to 
Table 7: Performance (HTER, in \%, lower is better) of ENet. Each row stands for the training dataset. Starting from the second column, each consecutive four columns form a group, and each group stands for the testing dataset. "NT" stands for normal training; "ETI" and "ET-G" stand for enhanced training with negative samples produced by unpaired linear interpolation (data-centric) and gradient-based distortion (model-centric), respectively; "MU" stands for "mixup" 35. The generalization performance results are in italics.

\begin{tabular}{|c|c|c|c|c|c|c|c|c|c|c|c|c|c|c|c|c|}
\hline \multirow{2}{*}{ Dataset } & \multicolumn{4}{|c|}{ Artlantis } & \multicolumn{4}{|c|}{ Autodesk } & \multicolumn{4}{|c|}{ Corona } & \multicolumn{4}{|c|}{ VRay } \\
\hline & NT & ET-I & ET-G & $\mathrm{MU}$ & NT & ET-I & ET-G & $\mathrm{MU}$ & NT & ET-I & ET-G & $\mathrm{MU}$ & NT & ET-I & ET-G & $\mathrm{MU}$ \\
\hline Artlantis & 1.31 & 2.31 & 2.75 & 1.75 & 10.06 & 5.09 & 4.31 & 11.95 & 14.58 & 8.15 & 7.28 & 16.03 & 11.86 & 7.22 & 5.50 & 11.86 \\
\hline Autodesk & 10.39 & $9 \quad 5.64$ & 5.58 & 13.30 & 1.56 & 3.08 & 2.39 & 1.70 & 7.67 & 5.39 & 4.86 & 9.44 & 13.39 & 7.47 & 8.22 & 14.44 \\
\hline Corona & 16.08 & 87.61 & 6.39 & 14.67 & 7.92 & 6.83 & 7.03 & 6.97 & 1.50 & 2.72 & 2.14 & 1.31 & 7.78 & 4.36 & 4.39 & 6.06 \\
\hline VRay & 11.58 & 6.94 & 5.39 & 9.22 & 9.97 & 5.97 & 6.08 & 9.72 & 4.53 & 4.31 & 3.17 & 4.33 & 1.25 & 2.22 & 1.72 & 1.19 \\
\hline
\end{tabular}

regularize the neural network and encourage the trained model to behave linearly in-between training examples. We train the ENet with "mixup" and set its hyperparameter $\alpha=0.4$ as recommended in [35]. All the results are reported in Table 7 Comparing the columns of "MU" with "ET-I" and "ET-G", we find that data-centric and model-centric based enhanced training significantly outperforms "mixup". Furthermore, the generalization performance of "mixup" sometimes is worse than that of normal training (without "mixup"), e.g., training on Autodesk and testing on Artlantis (10.39\% vs. 13.30\%). A possible reason is that "mixup" is essentially a form of data augmentation that implicitly affects the generalization of trained CNN model (in fact, this sometimes cannot guarantee the improvement of generalization as reported in Table 7 and mentioned above), whereas data-centric and model-centric based enhanced training can explicitly change the decision boundary and then improve the generalization of CNN-based detectors.

As shown above with experimental results, model-centric negative sample insertion usually achieves better performance in terms of conventional classification accuracy and generalization capability, especially for YaoNet, when compared with data-centric method. The reason is that model-centric method can more exactly control the location of negative samples relative to the decision boundary in the feature space of CNN, and thus more effectively improve the generalization with relatively small decrease of the classification accuracy on 


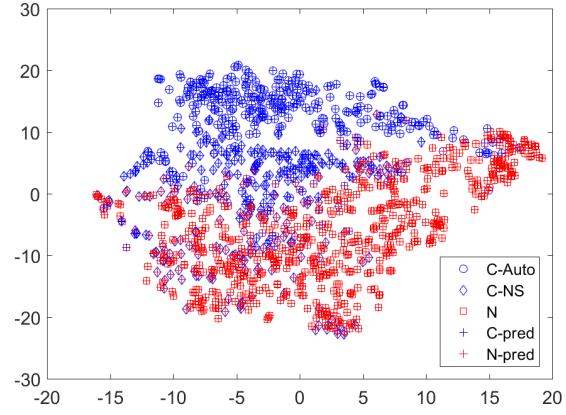

(a)

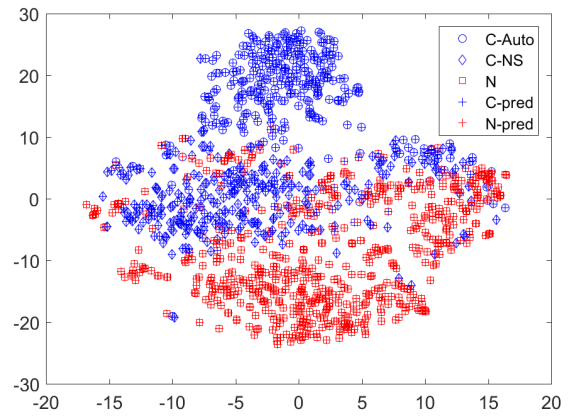

(b)

Figure 7: The deep feature visualization of YaoNet with t-SNE 36]. "C" means computergenerated images and "N" means natural images. "C-Auto" means the CG images rendered by Autodesk and "C-NS" means the negative samples generated by data-centric method (a) and model-centric method (b). "Y-pred" means that the predicted label of CNN is Y. NIs and CG images are randomly selected from training dataset for visualization.

NIs. To clearly illustrate the location of negative samples generated by datacentric and model-centric methods in the feature space, we train YaoNet on NIs and CG images rendered by Autodesk. We visualize the deep features of negative samples in the last insertion of enhanced training with t-SNE [36], and results are shown in Fig. 7. In Fig. 7(a), many negative samples are mixed with point cloud of NIs and predicted as NI (blue diamonds with red + ) because the linear interpolation is conducted in the image space and "blind" to CNN. On the contrary, all negative samples of model-centric method in Fig. 7)(b) are predicted as CG (blue diamonds with blue + ) and almost located in the middle of point clouds of NIs and CG images.

\subsection{Discussion}

In our study, we first observe a new problem regarding the generalization performance of CG forensics, then propose a new method to cope with this challenging problem, and finally validate the proposed method with extensive experiments. New understanding we get from this study is mainly the following: when we roughly know that a class may have a relatively large distribution 
change during testing, we can use our method to generate proxy samples of the "unknown" distributions by only using available training data; the enhanced training with such samples is effective to improve generalization. This is valid for the $12\left(4^{*} 3\right)$ tested cases. Our work is a small step towards the ultimate goal of fully understanding CNN's generalization. We have also tried to gain new understanding with FFT of first-layer filters and t-SNE visualization, which may provide useful insights to colleagues.

\section{Conclusion}

In this work, we studied and solved the challenging blind detection problem of CG image forensics. To facilitate this study, we collected four CG datasets with high level of photorealism. We designed and implemented a novel twobranch network with different initializations in their respective first layer to extract more diverse features, and this network has good generalization performance. In the meanwhile, we also introduced the data-centric and modelcentric negative sample generation used for conducting enhanced training. This can further improve the generalization performance of CNN-based detectors. More information and materials, including the source code, are available at https://github.com/weizequan/CGDetection.

For this new and challenging CG forensic problem, our method does not offer a rigorous framework/formulation, which can be considered as a limitation. However, this might also be an advantage: e.g., we avoid implicit restrictions due to mathematical modeling, such as specific hypothesized parameterization of the distributions of "unknown" CG images. Further, our approach does not use any sample or prior knowledge of new distributions, which is usually necessary for a rigorous formulation (e.g., in many domain adaptation algorithms). This makes our method simple, flexible and generic because our assumption is very weak and everything is done "off-line" at the training side. In the future, we plan to study the generalization improvement with a suitable rigorous formulation. Our proposed method and such a future method are not contradic- 
tory, and can even complement each other, e.g., our "off-line" method applied first, before an "on-line" continual learning method. Last but not least, our study implies that in order to improve generalization it is beneficial to learn diverse features and to learn from harder artificial samples. We would like to test this idea in other research problems and in the meanwhile explore other approaches to understanding and enhancing the generalization of CNN-based forensic detectors.

\section{Acknowledgment}

This work was funded in part by the National Natural Science Foundation of China (61772523 and 61620106003), the National Key R\&D Program of China (2019YFB2204104), the Beijing Natural Science Foundation (L182059), and the French National Agency for Research through PERSYVAL-lab under Grant ANR-11-LABX-0025-01 and through DEFALS under Grant ANR-16DEFA-0003. W. Quan acknowledges the support from the UCAS Joint PhD Training Program. We thank Dr. D.-T. Dang-Nguyen and Dr. D. Shullani for kindly sharing the RAISE [30] and VISION [31] dataset respectively. We also thank Leonardo Huspenina, Ezequiel Ribeiro, Sang Nguyen, Ahmad Ka-

mal, dartofang, Colin Langley, Nuno Silva, fobus, P\&M, cecofuli, and Caue Rodrigues, authors of the CG images shown in this paper.

\section{References}

[1] T. Yamaguchi, T. Yatagawa, Y. Tokuyoshi, S. Morishima, Real-time rendering of layered materials with anisotropic normal distributions, Computational Visual Media 6 (1) (2020) 29-36.

[2] Artlantis gallery, https://atl.artlantis.com/en/gallery/, (visited on 2020-03-11).

[3] Autodesk A360 rendering gallery, https://gallery.autodesk.com/ a360rendering/, (visited on 2020-03-11). 
[4] Corona renderer gallery, https://corona-renderer.com/gallery, (visited on 2020-03-11).

[5] Chaosgroup gallery, https://www.chaosgroup.com/gallery Learn VRay gallery, https://www.learnvray.com/fotogallery/, (visited on 2020-03-11).

[6] T.-T. Ng, S.-F. Chang, J. Hsu, L. Xie, M.-P. Tsui, Physics-motivated features for distinguishing photographic images and computer graphics, in: Proceedings of the ACM International Conference on Multimedia, 2005, pp. 239-248.

[7] S. Lyu, H. Farid, How realistic is photorealistic?, IEEE Transactions on Signal Processing 53 (2) (2005) 845-850.

[8] W. Chen, Y. Q. Shi, G. Xuan, Identifying computer graphics using HSV color model and statistical moments of characteristic functions, in: Proceedings of the IEEE International Conference on Multimedia \& Expo, 2007, pp. 1123-1126.

[9] A. C. Gallagher, T. Chen, Image authentication by detecting traces of demosaicing, in: Proceedings of the IEEE Conference on Computer Vision and Pattern Recognition, 2008, pp. 1-8.

[10] R. Zhang, R.-D. Wang, T.-T. Ng, Distinguishing photographic images and photorealistic computer graphics using visual vocabulary on local image edges, in: Proceedings of the International Workshop on Digital-Forensics and Watermarking, 2012, pp. 292-305.

[11] J. Wang, T. Li, Y.-Q. Shi, S. Lian, J. Ye, Forensics feature analysis in quaternion wavelet domain for distinguishing photographic images and computer graphics, Multimedia Tools and Applications 76 (22) (2017) $23721-23737$.

[12] F. Peng, D.-L. Zhou, M. Long, X.-M. Sun, Discrimination of natural images and computer generated graphics based on multi-fractal and regression 
analysis, AEU - International Journal of Electronics and Communications 71 (2017) $72-81$.

[13] N. Rahmouni, V. Nozick, J. Yamagishi, I. Echizen, Distinguishing computer graphics from natural images using convolution neural networks, in: Proceedings of the IEEE International Workshop on Information Forensics and Security, 2017, pp. 1-6.

[14] W. Quan, K. Wang, D.-M. Yan, X. Zhang, Distinguishing between natural and computer-generated images using convolutional neural networks, IEEE Transactions on Information Forensics and Security 13 (11) (2018) 27722787.

[15] Y. Yao, W. Hu, W. Zhang, T. Wu, Y. Q. Shi, Distinguishing computergenerated graphics from natural images based on sensor pattern noise and deep learning, Sensors 18 (4).

[16] P. He, X. Jiang, T. Sun, H. Li, Computer graphics identification combining convolutional and recurrent neural networks, IEEE Signal Processing Letters 25 (9) (2018) 1369-1373.

[17] H. H. Nguyen, J. Yamagishi, I. Echizen, Capsule-forensics: Using capsule networks to detect forged images and videos, in: Proceedings of the IEEE International Conference on Acoustics, Speech and Signal Processing, 2019, pp. 2307-2311.

[18] D. Bhalang Tarianga, P. Senguptab, A. Roy, R. Subhra Chakraborty, R. Naskar, Classification of computer generated and natural images based on efficient deep convolutional recurrent attention model, in: Proceedings of the IEEE Conference on Computer Vision and Pattern Recognition Workshops, 2019, pp. 146-152.

[19] R. Zhang, W. Quan, L. Fan, L. Hu, D.-M. Yan, Distinguishing computergenerated images from natural images using channel and pixel correlation, Journal of Computer Science and Technology 35 (3) (2020) 592-602. 
[20] B. Shuai, Z. Zuo, B. Wang, G. Wang, Dag-recurrent neural networks for scene labeling, in: Proceedings of the IEEE Conference on Computer Vision and Pattern Recognition, 2016, pp. 3620-3629.

[21] S. Sabour, N. Frosst, G. E. Hinton, Dynamic routing between capsules, in: Proceedings of the Advances in Neural Information Processing Systems, 2017, pp. 3856-3866.

[22] W. Quan, K. Wang, D.-M. Yan, D. Pellerin, X. Zhang, Improving the generalization of colorized image detection with enhanced training of CNN, in: Proceedings of the International Symposium on Image and Signal Processing and Analysis, 2019, pp. 246-252.

[23] N. Akhtar, A. Mian, Threat of adversarial attacks on deep learning in computer vision: A survey, IEEE Access 6 (2018) 14410-14430.

[24] X. Yuan, P. He, Q. Zhu, X. Li, Adversarial examples: Attacks and defenses for deep learning, IEEE Transactions on Neural Networks and Learning Systems 30 (9) (2019) 2805-2824.

[25] C. Szegedy, W. Zaremba, I. Sutskever, J. Bruna, D. Erhan, I. Goodfellow, R. Fergus, Intriguing properties of neural networks, in: International Conference on Learning Representations, 2014.

[26] I. Goodfellow, J. Shlens, C. Szegedy, Explaining and harnessing adversarial examples, in: International Conference on Learning Representations, 2015.

[27] A. Kurakin, I. J. Goodfellow, S. Bengio, Adversarial examples in the physical world, CoRR abs/1607.02533.

[28] F. Tramèr, A. Kurakin, N. Papernot, I. Goodfellow, D. Boneh, P. McDaniel, Ensemble adversarial training: Attacks and defenses, in: International Conference on Learning Representations, 2018.

[29] B. Tondi, Pixel-domain adversarial examples against CNN-based manipulation detectors, Electronics Letters 54 (21) (2018) 1220-1222. 
[30] D.-T. Dang-Nguyen, C. Pasquini, V. Conotter, G. Boato, RAISE: A raw images dataset for digital image forensics, in: Proceedings of the ACM Multimedia Systems Conference, 2015, pp. 219-224.

[31] D. Shullani, M. Fontani, M. Iuliani, O. A. Shaya, A. Piva, VISION: a video and image dataset for source identification, EURASIP Journal on Information Security 2017 (1) (2017) 15.

[32] W. Quan, K. Wang, D.-M. Yan, D. Pellerin, X. Zhang, Impact of data preparation and CNN's first layer on performance of image forensics: A case study of detecting colorized images, in: Proceedings of the IEEE/WIC/ACM International Conference on Web Intelligence - Companion Volume, 2019, pp. 127-131.

[33] J. Fridrich, J. Kodovský, Rich models for steganalysis of digital images, IEEE Transactions on Information Forensics and Security 7 (3) (2012) 868882.

[34] A. Krizhevsky, I. Sutskever, G. E. Hinton, ImageNet classification with deep convolutional neural networks, in: Proceedings of the Advances in Neural Information Processing Systems, 2012, pp. 1097-1105.

[35] H. Zhang, M. Cisse, Y. N. Dauphin, D. Lopez-Paz, mixup: Beyond empirical risk minimization, in: Proceedings of the International Conference on Learning Representations, 2018.

[36] L. van der Maaten, G. E. Hinton, Visualizing high-dimensional data using t-SNE, Journal of Machine Learning Research 9 (2008) 2579-2605. 